\title{
Medical Facelifts by Chemical Myoplasty, Myotension and Myopexy (Endopeel Techniques or Muscular SMAS Repositioning)
}

Alain Tenenbaum*

Facial Plastic Surgeon, Switzerland

\begin{abstract}
The immediate non invasive true medical face lifts using muscular repositioning without volume augmentation by myoplasty, myopexy and myotension (called Endopeel techniques) is a new weapon for facial plastic surgeons as cosmetic dermatologists.
\end{abstract}

A true facelift is a technique leading to a skin excess which will be removed, to perform a good skin tension without exaggeration (to avoid necrosis), and that without any volume augmentation.

Keywords: Endopeel techniques; Face lifts; Facial paralysis

\section{Introduction}

The Endopeel techniques can lead if decided by the facial plastic surgeon to a pretragal skin excess, which can be removed, and that too without volume augmentation. These techniques can be proposed to patients wishing to maintain the benefits of a surgical face lift, to patients which are not candidates for the surgery, to surgeons who want to complete their surgical operation acting on the forbidden areas for the surgery (terminal ramifications of the facial nerve) and/or the areas which are not reachable form in invasive surgery [1]. The advantages of these techniques are important for the patients: the immediate effect, the absence of scars, the absence of down time, the absence of social eviction, as the transitory complications which are limited only to short duration edema and ecchymosis which may be avoided with the use of some medications. Facial paralysis can benefit too of these techniques to decrease the social aspect offacial asymmetry.

\section{Warning about so called "Medical facelifts"}

Fillers which just increase the volume, mesotherapic products as botulinum have nothing to do with facelifts, as they never provoke a tension effect without volume augmentation leading to a skin excess, which will be removed.

Actually the true facelifts can be realized or by surgery, or by Endopeel techniques, if we respect the first facelift definition.

\section{Benefits and advantages for the facial plastic surgeon and/or the cosmetic dermatologist}

- To perform a scar less true facelift with an immediate result, without using volumetric medical devices or paralyzing medicine.

- To perform an immediate facelift without undermining leading to a pretragal skin excess which can be removed easily or which can be hidden with the Aptos threads which will help to make an appropriate repartition of this skin excess, socially acceptable.

- To give back the lost tensionto muscles involved in a facial paralysis.

\section{Techniques chosen}

- Intramuscular trivectorial injections using the surgical logic of a facelift, will be exposed requiring a basic anatomy physiological knowledgeto realize anasal labial furrow lifting on one side and to lift the sadly baggy low jowls on another sideas to make a recon touring of the mandible.

- A special protocol allows also getting a pre tragal skin excess which can be removed as a dog ear excision or with the Aptos threads scar less technique, with a certain amount of Endopeel carbolic acid. This interesting strategy allows a long duration of Endopeel effect keeping a mini invasive lifting technique using the criss cross technique with a multiholes $2.0 \mathrm{~mm}$ cannula.

\section{Patients selection}

- All patients who desire a surgical or a not surgical can be candidates, as those not being candidate for any kind of filler injected in the naso labial furrows (the fillers not only can give unaesthetic aspects called rolling bulges, but are too often sources of complications like granulomas, necrosis, migration, nodules etc.), just as those which have the first semiological signs leading to the right indications of face lifts [2].

- Patients with irreversible facial paralysis are good candidates for Endopeel techniques which will give them less asymmetry (static as dynamic) and restituting them almost their social appearance prior to the facial paralysis event.

- Our criteria of exclusion are: exaggerate alcoholic patients, heavy smokers, pregnant women, subjects under 18 years, patients known with allergy to peanuts or those having antecedents mentioning a Quincke edema, as patients affected with a cardiopathy, nephropathy and liver problems. All the patients without any exception when the outside temperature exceeds $40^{\circ} \mathrm{C}$ are also excluded, because edema scan last for 1 week but regress under corticotherapy if this one is given right before beginning the procedure.

*Corresponding author: Alain Tenenbaum, Facial Plastic Surgeon, Vergio $\mathrm{Nr}$ 37, CH-6932Lugano, Switzerland, Tel: +41-764177315; E-mail: contact@facialplasticsurgery.org, drpeeling@aol.com

Received June 10, 2013; Accepted July 17, 2013; Published July 24, 2013

Citation: Tenenbaum A (2013) Medical Facelifts by Chemical Myoplasty, Myotension and Myopexy (Endopeel Techniques or Muscular SMAS Repositioning). Anaplastology 2: 116. doi: 10.4172/2161-1173.1000116

Copyright: $\odot 2013$ Tenenbaum A. This is an open-access article distributed under the terms of the Creative Commons Attribution License, which permits unrestricted use, distribution, and reproduction in any medium, provided the original author and source are credited. 
- The ideal candidate is not alcoholic nor smoker without any muscular atrophy, without treatments using botulinum toxin or fillers since 3 months, in apparent good health, with an age range between 20 and 85 years, neither fat nor too thin, eager of immediate face lift without surgery and scars.

\section{Benefits and advantages envisaged for the patients}

- The face lift effect is immediate and is perfectly visible 30 minutes after the end of the treatment with a maximum result 2 to 3 days after the procedure.

- The face lift effect is early and will be relayed by the late peeling effect, specific to the techniques Endopeel.

- The treatment can be ineffective in $8 \%$ of the cases (bad technique).

- The effectiveness of this treatment increases with the number or the repetition of the procedures because the basic muscular tone will be thus increasingly high on the scale of the tension of this last.

- It is easy to prove objectively the effectiveness of this treatment by treating, for example, only one half face on the same patient and by making a comparison between the treated half face and the controlateral untreated one by pure visual inspection, or by photographic images, or by using cephalometric points of reference.

- These same parameters are taken again to evaluate the duration of the results objectively.

- The procedure is made comfortable for the pusillanimous patients thanks to the special anaesthetic gel (Tenenbaum anesthetic lipogel) applied before the Endopeel procedure and giving satisfaction of painless technique to each patient, because the protocol can be adapted perfectly according to the timetable of the patient and also according to his social requirements.

- In the field of safety, so far and since 16 years these techniques started to be used, no legal procedure against a physician using the techniques Endopeel was announced and/or indexed to our knowledge. The only complications met are only transitory, like edema ecchymosis which never exceeds 10 days in worst cases without medications.

- The report/ratio benefit cost of the treatment is accepted in the large majority of the cases.

- Less asymmetry and none social eviction for patients affected with facial paralysis.

\section{Techniques}

- The selection of the patients has been described above.

- The material to be used is the following :

- The medical device like oily carbolic acid (DocTNB $\left.{ }^{\circ}\right)$ completely different from phenol (chemical differences and analogies will be detailed with their consequences).

- Syringes $1 \mathrm{ml}$ luer lock or a multihole canula $2.0 \mathrm{~mm}$.

- Flexible needles for the face $30 \mathrm{~g}$.

- 1 needle $18 \mathrm{~g}$ to aspire the solution of oily carbolic acid.
- Ananesthetic specific gel containing lidocaine $23 \%$, tetracaine $7 \%$, using an emollient lipophilic lubricating gel like vector.

- A post, Endopeel, cold cream.

- Finally one not alcoholic disinfecting product, gloves and gauzes.

- The technique known as standard treats 7 areas: the malar area, the zygomatic arc, the masseter area, the area of the $1 / 3$ inferior of the face, the mandible edge, the angle of the mandible, the cervical area being above the higher limit of the cricoidor thyroid cartilage.

- The technique to lift the nasallabial furrow will involve particularly the 8 following muscles: major, and minor zygomatic, risorius, buccinators, depressor of the angle of the mouth, elevator of the angle of the mouth, elevatorof the upper lip and of the nasal aisle.

- The technique for sadly low jowls cancellation involves the following muscles: platysma, digastric, mylo hyoïdeus, buccinators, depressors of the angle of the mouth.

- These 2 last techniques are rather reserved to the facial plastic surgeons, because they are not only one complement of the traditional surgery of the face lift while intervening on the zone of the terminal ramifications of the facial nerve (danger zone for the surgery), but also allow maintenance of a surgical face lift while intervening on the facial muscles by myopexy and myoplasty.

- The basic technique consists in injecting perpendicular to the plan of the muscle $0.05 \mathrm{ml}$ of oilycarbolic acid each $\mathrm{cm}$ and same in the perpendicular plan to the precedent following the direction and the sense to obtain the wished deformation of the muscle using criss cross-technique.

\section{Errors}

The most current errors are not the IM injections made in SC (subcutaneous) which will give the desired effect (because the oily product will diffuse from SC tissue to the muscle) will use more quantity of product and will be responsible of an important edema not appreciated by the patients, which can last between 3 and 8 days without medication. Another error consists in touching the periosteum with the injection needle, which will provoke ecchymosis which can be hidden thanks to a makeup and which will disappear from 3 to 8 days without discomfort for the patient. The intra vascular injection of Endopeel carbolic acid cannot be regarded as an error, considering the sclerosing effect of this substance. The injection into the motor plate or any nerve of carbolic acidis known for the treatment of the trigeminal neuralgias and may slow down the speed of nervous conduction for one short duration.

\section{Side effects, complications and alternative treatments}

The side effects or complications are only transitory and are summarized with an early edema and some ecchymosis easily treated and being able to be avoided. As for late edema occurring at the 10th day after the procedure, it is related to an allergy to peanuts and is treated only with antihistaminic. $2 \%$ of the Asian population is allergic to peanuts. A preliminary test is useless. Actually there is no alternative to the myotension, myopexy and myoplasty using other products and techniques than Endopeel. But the Endopeel techniques can be potentialized using chemical peelings as other existing treatments 
Citation: Tenenbaum A (2013) Medical Facelifts by Chemical Myoplasty, Myotension and Myopexy (Endopeel Techniques or Muscular SMAS Repositioning). Anaplastology 2: 116. doi: 10.4172/2161-1173.1000116

Page 3 of 3

in medicine and cosmetic surgery (skin tightening effect with radio frequency $4 \mathrm{MHz}$ ).

\section{Research and applications}

A study on cadavers masseter made by the author, demonstrates that there is no mass and/or volume augmentation between the treated masseter and the untreated one. But all semiological rejuvenation effects could be seen immediately on the half face treated after few minutes. After dissection, the aspect of the treated masseter was similar to a young alive masseter with a nice color and with good tension compared to the untreated masseter looking atrophic, without vitality without any tension and with cadaveric color. Also some recent experimentation demonstrate that, Endopeel, works also on very thin muscles, even in absence of nervous signals.

\section{Conclusion}

Until now, the concept of facial rejuvenation was based only on volume augmentation concept (fillers) as myorelaxation with myoatrophy (botulinum toxin), from now and thanks to the Endopeel techniques one acts not only on the myotension by preserving the muscular mass and without affecting the muscular contraction but also on the myoplasty and the myopexy. With this fact the indications of the fillers become more restricted and will apply only to the depressions which remain after tissue tension of the selected area. Finally chemical true face lifts are not due as much of manufacturers claim it with an increase in volume by fillers but rather with a handing-over in tension of tissues with tightening effect like the Endopeel techniques. The techniques of Endopeel are thus a new strategic weapon for the facial plastic surgeons enabling them to complete and/or maintain a face liftand to propose an alternative to the surgery.

\section{References}

1. Tenenbaum A (2009) La tecnicaEndopeel- La medicinaestetica-A.Redaelli, EEAFlorence.

2. Dewandre L,Tenenbaum A (2011) The Chemistry of Peels ( $2^{\text {nd }}$ edn), Saunders Elsevier. 